\title{
A novel image de-noising method based on spherical coordinates system
}

Degan Zhang ${ }^{1,2}$, Xuejing Kang ${ }^{1,2^{*}}$ and Jinghui Wang ${ }^{1,2}$

\begin{abstract}
In this article, a novel image de-noising method is proposed. This method is based on spherical coordinates system. First, spherical transform is re-defined in wavelet domain, and the properties of the spherical transform in wavelet domain are listed. Then, a new adaptive threshold in spherical coordinate system is presented. It has been proved based on Besov space norm theory. After that, a novel curve shrinkage function is proposed to overcome the limitation of the traditional shrinkage functions. The new function can reach and exceed the true value and enhance the edge of the image. Finally, the multi-scale product in wavelet domain is introduced to spherical coordinates system. This article names the multi-scale product in spherical coordinates system as Multi-Scale Norm Product. The experimental results compared the improved algorithm with other methods from the peak signal-tonoise ratio, mean square error, and running time. The results indicate that improved algorithm is simple and effective.
\end{abstract}

Keywords: wavelet translation, image de-noising, multi-scale product, shrinkage function, spherical coordinates.

\section{Introduction}

Noise produced in image acquisition and transmission will cause image quality decline. Therefore, image denoising is an important problem in the field of image processing [1-4]. The goal of image de-noising is to recover the original image from such a noisy copy. The traditional de-noising methods cause details blurring of the reconstructed image. Discrete wavelet transform (DWT) provides a fast, local, sparse, and decorrelates multi-resolution analysis of signals. It can reconstruct high-quality images [5]. In recent years, wavelet shrinkage algorithm for image de-noising has widely been used in different fields [6-8].

The threshold and shrinkage function are the key factors in image de-noising. They will determine the effectiveness of image de-noising. Donoho $[9,10]$ presented a method named wavelet shrinkage and showed its obvious efficiency on signal de-noising. They proved that wavelet shrinkage was nearly optimal over a wide rage of function classes and error criterions. They also provided an estimated function with smoothness not

\footnotetext{
* Correspondence: xuejing.nuli@126.com

'Key Laboratory of Computer Vision and System, Tianjin University of

Technology, Ministry of Education, Tianjin 300384, China

Full list of author information is available at the end of the article
}

less than the original. With these advantages, the method began to be widely applied. However, the universal threshold $T=\sigma \sqrt{2 \ln (N * M)}$ tended to set all the coefficients of image details to zero, especially when $N$ approaches infinite. At the mean while, the standard deviation of noise must be pre-estimated. Bias existed between estimated value and real value will reduce the effect of image de-noising. DeVore [11] and Feng et al. [12] believe the image space belongs to Besov space. In 1998, Chambolle et al. [13] proposed a threshold in Besov space.

The classic shrinkage functions are the hard and soft threshold functions $[9,10]$. Many researchers have proposed improved methods to optimize the classic algorithm. Khare et al. [14] proposed a multi-scale adaptive soft-threshold function and achieved a good de-noising effect. However, in this method, the wavelet coefficients were processed directly. It will cause distortion of the reconstructed image. Wang [15] introduced a hyperbolic shrinkage function. This function avoided processing the wavelet coefficients directly. However, the hyperbolic function could not achieve the true value of the coefficients and cause edge blurring. Li et al. [16] presented an improved soft-threshold function. This function is more flexible than the classic soft and hard thresholds. 
Table 1 De-noising effects evaluation of different decomposing level

\begin{tabular}{lllll}
\hline & Level & PSNR & MSE & Time \\
\hline Lena & 1 & 28.0877 & 100.9974 & 0.7438 \\
& 2 & 29.6917 & 69.8088 & 0.8150 \\
& 3 & 30.1217 & 63.2281 & 1.1094 \\
& 4 & 30.1198 & 63.2558 & 1.8175 \\
& 5 & 30.1198 & 63.2558 & 2.5694 \\
IOT & 6 & 27.2008 & 123.8798 & 3.8712 \\
& 1 & 27.6682 & 111.2398 & 0.6987 \\
& 2 & 28.4816 & 92.2402 & 0.8150 \\
& 3 & 29.6977 & 69.1725 & 1.2526 \\
& 4 & 29.6977 & 69.1725 & 1.8725 \\
& 5 & 29.7011 & 69.6579 & 2.5694 \\
& 6 & 25.5632 & 180.6177 & 4.0925 \\
\hline
\end{tabular}

However, the starting point of this function is origin. There will be some noise information mistakenly processed as signal information.

Bao and Zhang [17] and Meng et al. [18] used multiscale products to control the shrinkage of the wavelet coefficients. Multi-scale products can fully use the correlation of wavelet coefficients in different scales. Inspired by this idea, multi-scale norm product (MSNP) is defined in this article to control the shrinkage of the wavelet coefficients.

This article proposes a new threshold after analyzing the relationship between wavelet coefficients norm, Besov space norm, and radial component in the spherical coordinates, and a new curve shrinkage function is proposed to achieve good de-noising effect. The coefficients estimated by new function can reach and exceed the real value. It will avoid the edge blurring and enhance the coefficients of contours. Experimental results show that the improved method in this article retains the advantage of spherical coordinates. This new method cannot only be provided with effectively denoising, but also have better preserve image details and enhance the image edge information.

\section{Traditional wavelet shrinkage algorithm}

Suppose that it is desired to recover an unknown signal $f(x, y)$ from a noise image $f *(x, y)$,

$$
f^{*}(x, y)=f(x, y)+\sigma \varepsilon_{i}
$$

where $\varepsilon_{i}$ is independent and identically distributed (i.i. d) as $N(0,1), \sigma$ refers to the noise level.

The idea of the wavelet shrinkage de-noising is large wavelet coefficients reserve the useful information of image, while smaller coefficients contain the noise information which should be abandoned. The steps of the wavelet de-noising algorithm are
- To decompose noising image in wavelet domain; - Use threshold $T$ and shrinkage function to process the high-frequency coefficients.

Hard-threshold function:

$$
w^{*}(x, y)= \begin{cases}w(x, y) & w(x, y) \geq T \\ 0 & w(x, y)<T\end{cases}
$$

Soft-threshold function:

$$
w^{*}(x, y)= \begin{cases}\operatorname{sign}(w(x, y)) * w(x, y) & w(x, y) \geq T \\ 0 & w(x, y)<T\end{cases}
$$

where $w(x, y)$ denotes wavelet coefficients, $w^{*}(x, y)$ is wavelet coefficients after processing, $T$ is the threshold, $\operatorname{sign}(\cdot)$ is sign function.

- To process the low-frequency and high-frequency coefficients by inverse wavelet transform and reconstruct the image.

\section{Spherical transform re-defined in wavelet domain}

Skowronski [19] firstly introduced spherical coordinates system into wavelet domain. Wang [15] extended this idea. However, he did not propose the properties of spherical transform in wavelet domain, neither define a proper threshold in spherical coordinates system.

This article re-defines the spherical transform in wavelet domain and lists its properties. Set $f$ denotes a twodimensional image, $C_{j, k}^{(i)}, i=1,2,3$ is the high-frequency parts of the image $f$ after wavelet decomposing.

Define 1: The spherical transform in wavelet domain is defined as follows:

$$
\left\{\begin{array}{l}
R=\sqrt{\left(C_{j, k}^{(1)}\right)^{2}+\left(C_{j, k}^{(2)}\right)^{2}+\left(C_{j, k}^{(3)}\right)^{2}} \\
\theta=\operatorname{tg}^{-1}\left(C_{j, k}^{(2)} / C_{j, k}^{(1)}\right) \\
\varphi=\cos ^{-1}\left(C_{j, k}^{(3)} / \sqrt{\left(C_{j, k}^{(1)}\right)^{2}+\left(C_{j, k}^{(2)}\right)^{2}+\left(C_{j, k}^{(3)}\right)^{2}}\right)
\end{array}\right.
$$

where $C_{j, k}^{(1)}, C_{j, k}^{(2)}, C_{j, k}^{(3)}$, respectively, represent horizontal, vertical, and diagonal components. $R$ is the radial component in spherical coordinate system, $\theta, \phi$ are the phase angle components. Figure 1 shows the correspondence relationship of the components between spherical coordinate system and wavelet domain.

Skowronski [19] only expressed the Haar coefficients in spherical coordinates and the radial component came from the variance of a block. Compared with the define of Skowronski, in this article, the wavelet coefficients 


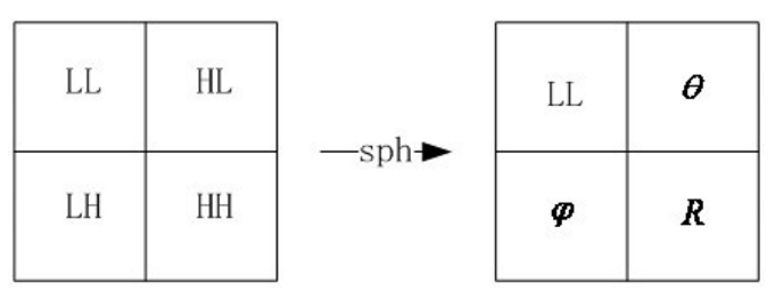

Figure 1 Spherical transformation in wavelet domain

are mapped to the components in spherical coordinate system. This define is reversible and exactly establishes one-to-one correspondence between wavelet coefficients and components in spherical coordinate system. The properties of spherical transform in wavelet domain lists as follows:

Property 1: The image energy in the three high-frequency parts is completely mapped to radial component $R$ of spherical coordinate system. The phase angle components $\theta, \phi$ only contain the location information.

From Property 1, the energy in high-frequency parts is completely mapped to radial component. Therefore, we just need to process the radial component in spherical coordinate system. This idea can avoid processing the wavelet coefficients directly. It reduces the image distortion in a certain extent.

Property 2: Radial component $R$ and the phase angle components $\theta, \phi$ are mutually independent. The small angle does not always correspond to little energy.

Property 3: Spherical transform can further reduce the correlation among coefficients in the same scale.

Property 4: Spherical transform does not change the correlation among coefficients in different scales.

Figure 2 demonstrates Properties 3 and 4 .

\section{A novel threshold in spherical coordinates}

DeVore [11] and Feng et al. [12] have proved the image space belong to Besov space. Chambolle et al. [13] proposed a threshold in Besov space. Based on Chambolle threshold, this article presents a novel shrinkage threshold which is optimal in spherical coordinates. The new threshold does not require pre-estimated noise intensity.
It avoids the incomplete de-noising problem caused by inaccurate estimates.

Theorem 1: The threshold in spherical coordinates system is

$$
T=\sqrt{6}|R| / N
$$

where $N$ denotes image pixel, $R$ is radial component in spherical coordinates.

Proof: Chambolle et al. [13] proposed a better shrinkage threshold in Besov space as follows:

$$
N \leq T^{-q}\|F\|_{B_{q}^{\beta}\left(L_{p}(\Omega)\right)}^{q}
$$

where $N$ is the number of image pixel, $R$ is radial component in spherical coordinates system. The equivalent relationship between Besov space norm $\|F\|_{B_{q}^{\beta}\left(L_{p}(\Omega)\right)}$ and radial component norm is established in three steps.

(a) First, to establish equivalent relationship between Besov space norm $\|F\|_{B_{q}^{\beta}\left(L_{p}(\Omega)\right)}$ and wavelet coefficients norm $\left|C_{j, k}^{(i)}\right|, \quad i=1,2,3$.

Suppose $f$ is a noise image in the interval $\Omega=[0,1)^{2}$. It is proper to use Besov space norm to describe the regularity of image $[11,12]$. First, the image $f$ is decomposed by compactly supported orthogonal wavelet Daubechies (db2) in this article. Suppose $\phi_{j, k}=1$ denotes a cycle scaling function. $\Psi=\left\{\psi^{(1)}, \psi^{(2)}, \psi^{(3)}\right\}$ is a set of

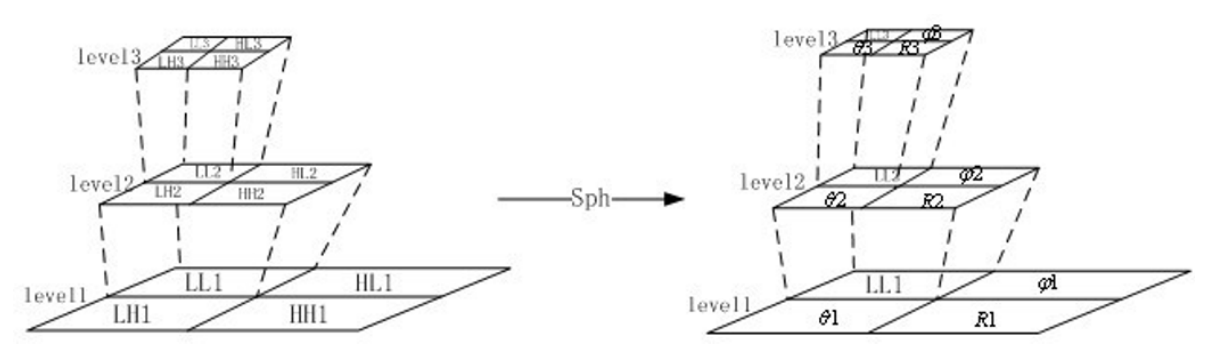

Figure 2 Multi-scale spherical transformation. 
orthogonal wavelet bases in the space $W_{j}^{2}$, where

$$
\begin{aligned}
& \psi_{j, k}^{(1)}\left(x_{1}, x_{2}\right)=\psi\left(x_{1}\right) \varphi\left(x_{2}\right) \\
& \psi_{j, k}^{(2)}\left(x_{1}, x_{2}\right)=\varphi\left(x_{1}\right) \psi\left(x_{2}\right) \\
& \psi_{j, k}^{3}\left(x_{1}, x_{2}\right)=\psi\left(x_{1}\right) \psi\left(x_{2}\right)
\end{aligned}
$$

the 2D-DWT of image $f$ is

$$
f=\sum_{j \in Z^{2}, k \in K} C_{j, k}^{(i)} \Psi_{j, k}+\sum_{j \in Z^{2}, k \in K} d_{j, K} \varphi_{j, K} i=1,2,3
$$

where $C_{j, k}^{(i)}=<f, \psi_{j, k}^{(i)}>, d_{j, k}=<f, \varphi_{j, k}>$, for $\phi_{j, k}=1$, the formula (8) can be rewritten as

$$
f=\sum_{j \in Z^{2}, k \in K} C_{j, k}^{(i)} \Psi_{j, k}+<f, 1>i=1,2,3
$$

Use wavelet coefficients norm to represent the norm in the space $L_{p}(\Omega)$.

$$
\|f\|_{L_{p}(\Omega)}^{p}=\sum_{j \in Z^{2}, k \in K}\left|C_{j, k}^{(i)}\right|^{p}+|<f, 1>|^{p}
$$

If wavelet coefficients $\psi_{j, k}^{(i)}(i=1,2,3)$ belong to the space $B_{p}^{\beta}\left(L_{p}(\Omega)\right), \beta>\alpha$

$$
\|f\|_{B_{p}^{\alpha}\left(L_{p}(\Omega)\right)} \approx\left(\sum_{j \in Z^{2}, k \in K} 2^{\alpha k p} 2^{k(p-2)}\left|C_{j, k}^{(i)}\right|^{p}\right)^{1 / p}
$$

As a general rule, we just need to consider the space $B_{1}^{\alpha}\left(L_{1}(\Omega)\right)$, here $p=1, \alpha \leq 1, k \geq 0$, then

$$
\|f\|_{B_{1}^{\alpha}\left(L_{1}(\Omega)\right)} \approx \sum_{k \in K, j \in Z^{2}} 2^{k(\alpha-1)}\left|C_{j, k}^{(i)}\right|
$$

Thus, the equivalent relationship between Besov space norm and wavelet coefficients norm has been established.

(b) Second, to establish equivalent relationship between the threshold $T$ in Besov space and wavelet coefficients norm $\left|C_{j, k}^{(i)}\right|, i=1,2,3$.

Set $q=1$, shrinkage threshold formula (6) can be rewritten as follows:

$$
T \leq\|F\|_{B_{1}^{\alpha}\left(L_{1}(\Omega)\right)} / N
$$

Therefore,

$$
T \leq \sum_{k \in K, j \in Z^{2}} 2^{k(\alpha-1)}\left|C_{j, k}^{(i)}\right| / N
$$

Generally, dyadic wavelet is used to deal with discrete image signal, for $\alpha \leq 1$, set $\alpha=\frac{1}{2}$, the formula (14) can be rewritten as follows

$$
T \leq \sum_{k \in K, j \in Z^{2}} 2^{-k / 2}\left|C_{j, k}^{(i)}\right| / N
$$

According to the property of inner product

$$
2^{-k / 2} C_{j, k}^{(i)}=2^{-k / 2}<f, \psi_{j, k}^{(i)}>=<f, 2^{-k / 2} \psi_{j, k}^{(i)}>
$$

Based on double scaling equation of wavelet, the following formula will be established:

$$
\begin{aligned}
2^{-k / 2} \psi_{j, k}^{(i)} & =2^{-k / 2} \psi\left(2^{-k} x-k\right) \\
& =2^{-k / 2} \sqrt{2} \sum_{n \in Z} g_{n} \varphi\left(2^{-k+1} x-2 j-n\right) \\
& =\sum_{n \in Z} g_{n} \varphi_{j-1,2 k+n}(x)=\sum_{n \in Z} g_{n-2 k} \varphi_{j-1, n}(x) \\
& =\psi_{j-1, k}^{(i)}
\end{aligned}
$$

Therefore,

$$
2^{-k / 2} C_{j, k}^{(i)}=2^{-k / 2}<f, \psi_{j, k}^{(i)}>=<f, \psi_{j-1, k}^{(i)}>=C_{j-1, k}^{(i)}
$$

From the formulas (15) and (18), shrinkage threshold in wavelet field is

$$
T \leq \sum_{k \in Z, j \in Z^{2}}\left|C_{j-1, k}^{(i)}\right| / N \quad i=1,2,3
$$

(c) Finally, to establish equivalent relationship between wavelet coefficients norm $\left|C_{j-1, k}^{(i)}\right|$ and radial component norm $|R|$ in spherical coordinates system.

The noise image $f$ is decomposed on multi-scale wavelet in this article. The high-frequency parts $C_{j-1, k^{\prime}}^{(i)} \quad i=1,2,3$ are mapped to $\theta, \phi$ and radial component $R$ in spherical coordinates system. Based on 
Properties 1 and 2, threshold $T$ just needs to process radial component $R$. So, the equivalent relationship between radial component $R$ and high-frequency $C_{j-1, k}^{(i)}$ needs to be established.

Based on the spherical transform in wavelet domain (formula 4), the following inequality will be established:

$$
\left\{\begin{array}{l}
R^{2}>\min \left\{\left(C_{j-1, k}^{(i)}\right)^{2}\right\}+\max \left\{\left(C_{j-1, k}^{(i)}\right)^{2}\right\} \\
R^{2} \geq 3 \min \left\{\left(C_{j-1, k}^{(i)}\right)^{2}\right\}
\end{array}\right.
$$

Therefore,

$$
\frac{2}{3} R^{2} \geq \max \left\{\left(C_{j-1, k}^{(i)}\right)^{2}\right\} \geq\left(C_{j-1, k}^{(i)}\right)^{2}
$$

where $i=1,2,3 k \geq 0, j \in\left\{0,1, \ldots .2^{k}-1\right\}^{2}$.

Thence, the following relationship will be established between wavelet coefficients norm and radial component norm.

$$
\left|C_{j-1, k}^{(i)}\right| \leq \sqrt{2 / 3}|R|
$$

The interval of shrinkage threshold will be

$$
T \leq \sqrt{6}|R| / N
$$

The least upper bound of $T$ is chosen in this article as the final shrinkage threshold:

$$
T=\sqrt{6}|R| / N
$$

where $N$ denotes the number of image pixel.

Many studies have demonstrated that different waveletdecomposed scales play an important role in image process. The de-noising effect will be improved if we choose the proper decomposed scales. As everyone knows, noise information is especially rich in high-frequency region. At different scales, noise information has different distribution characteristics. Therefore, different shrinkage thresholds need to be chosen at different scales. After analyzing wavelet multi-scale characteristics, the threshold at different scales is multiplied by scaling factor $\eta_{k}=$ $1 / 4^{k-1}$. Therefore, the shrinkage threshold under different scales is finally determined as follows:

$$
T_{k}=\eta_{k}\left(\sqrt{6}\left|R_{k}\right| / N\right), k=1,2,3, \ldots
$$

Based on the above proof, the new threshold has the following advantages:

(1) After the third or more decomposition of wavelet transform, the scaling factor is $\eta_{k} \leq \frac{1}{16}$, therefore, it can simplify the process and advance the operation speed.

(2) Do not need to estimate the standard deviation of noise before image de-noising, which will avoid the error existed between estimated value and real value and reduce the complexity of the algorithm.

\section{MSNP in spherical coordinates system}

In the wavelet domain, there is a correlation among the coefficients in different scales. If the amplitude of coefficients in small scales is large, at a big probability, the amplitude of coefficients in corresponding big scales is large too. Multi-scale product is based on this property of wavelet transform. Inspired by the idea of multi-scale product used in image de-noising $[15,16]$, this article defines an MSNP which is based on the following premises.

(1) In the wavelet domain, with the increase of decomposition level, the wavelet coefficients of effective signal will increase or remain unchanged. While the wavelet coefficients of noise signal will decrease.

(2) Spherical transform reduces the relevance of scaling interior. However, it does not change the correlation of wavelet coefficients in different scales (Figure 2).

Define 2: The MSNP at the point $(x, y)$ in spherical coordinates system is defined as follows:

$$
p_{2^{k}}(x, y)=\prod_{j=1}^{K} R_{j}(x, y)
$$

where $K$ is the decomposing scales. The MSNP has the following properties:

Property 5: The MSNP in spherical coordinates must be positive value.

Property 6: "The larger (effective signal) the greater, the smaller (noise signal) the smaller". With the increase of the decomposition level $K$, we will get the conclusion $p_{2^{k}}($ signal $)>>p_{2^{k}}$ (noise) .

The MSNP is controlled by the new threshold proposed in Section 4 in the image de-noising. It can enlarge the distance between effective signal and noise signal. It avoids the disadvantage of the traditional methods that smaller wavelet coefficients are wrongly removed. Therefore, MSNP will effectively remove the additive noise without loss of the important features of the original image.

\section{A new curve shrinkage function in spherical coordinates system}

From the promise 1 mentioned in Section 5, the maximum amplitude wavelet coefficients must be the effective signal.

At present, the improve algorithms about shrinkage function are infinitely close to the true value. However, 
they cannot reach the true value, which will cause the edge blurry of reconstructed image.

In order to gain better de-noising effect, this article constructs a new curve shrinkage function. It can ensure that the estimated signal is continuous at the threshold, and with the signal amplitude increases, the estimated value can reach and exceed the true value. It not only avoids the edge blurry, but also enhances the critical information. The new curve shrinkage function is

$$
R^{*}(i, j)=\left\{\begin{array}{lr}
\rho \cdot\left(|R(i, j)|-(1+\log (1+|R(i, j)|-T))^{-1} \cdot T\right) & R(i, j) \geq T \\
0 & R(i, j)<T
\end{array}\right.
$$

where $\rho=1+\frac{1}{5 \pi}|\arctan (|R(i, j)|-T)|$ is the adjust factor of the curve shrinkage function, $N, M$ are the image dimensions, $i=1,2, \ldots, N, j=1,2, \ldots, M$.

The advantages of the improved curve shrinkage function are listed below.

(a) When $R(i, j)=T$, it is easy to verify $R^{*}(i, j)=0$. So, the new function is continues at $T$.

(b) When $R(i, j)>T$, the proposed function meets the following inequality:

$$
R(i, j)-T \leq|R(i, j)|-(1+\log (1+|R(i, j)|-T))^{-1} \cdot T \leq R(i, j)
$$

Therefore, the radial component $R^{*}(i, j)$ estimated by the new curve function is located between hard and soft thresholds.

(c) As $|R(i, j)|-(1+\log (1+|R(i, j)|-T))^{-1} \cdot T$ is nonlinearity function, and $\log (\cdot)$ is a monotone increasing function, when $R(i, j) \rightarrow \infty$, the conclusion $R^{*}(i, j) \rightarrow R$ $(i, j)$ will be established. Therefore, with the increase of $R(i, j)$, the new curve function will infinite approximate to true value.

(d) The adjust factor $\rho=1+\frac{1}{5 \pi}|\arctan (|R(i, j)|-T)|, \rho \in[1,1.1]$ in the new function will raise the approach speed of the formula $R^{*}(i, j) \rightarrow R(i, j)$. Arctan $(\cdot)$ is a monotone continuous function, which can ensure $R^{*}(i, j)$ smooth approach to $R(i, j) . \rho \in[1,1.1]$, as the signal amplitude increases, the estimated signal can reach and exceed the real value. Large radial component has a certain enhancement, and this increase is limited to $<110 \%$ of the original energy. Therefore, it can effectively preserve the edge of the signal, and avoid the local enhancement caused by the unexpected.

Figure 3 shows the contrast between different shrinkage functions. In Figure 3, soft and hard thresholds were presented by Donoho $[9,10]$, MATS Method was proposed by Khare et al. [14], Hyperbolic shrinkage was

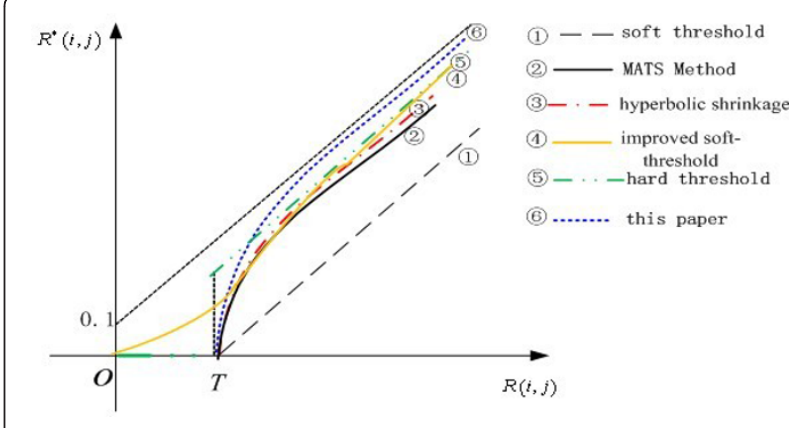

Figure 3 Different shrinkage function contrast diagram

used by Wang [15]. Li et al [16] presented the improved soft-threshold function.

\section{Algorithm design}

Suppose $f$ is the observed noise image. The steps of the improved algorithm:

Step 1. First, the image $f$ is decomposed by wavelet transform.

Step 2. The high frequencies $C_{j, k}^{(i)}, i=1,2,3$ of the image at different scales are transformed to spherical coordinates system by formula (4).

Step 3. Calculate the MSNP $p_{2^{k}}$

Step 4. Process the radial components $R_{k}$ at different scales

(a) At the first decomposition, the interference brought by noise is strong, the improved curve shrinkage function proposed in this article is used to process the radial components:

$R^{*}(i, j)=\left\{\begin{array}{lr}\rho \cdot\left(|R(i, j)|-\left(1+\log \left(1+|R(i, j)|-T_{k}\right)\right)^{-1} \cdot T_{k}\right) R(i, j) \geq T_{k} \\ 0 & R(i, j)<T_{k}\end{array}\right.$

(b) At the second or more decomposition of wavelet transform, the new threshold proposed in this article controls the MSNP for image de-noising.

$R^{*}(i, j)=\left\{\begin{array}{lr}\rho \cdot\left(|R(i, j)|-\left(1+\log \left(1+|R(i, j)|-T_{k}\right)\right)^{-1} \cdot T_{k}\right) & p_{2^{k}}(i, j) \geq T_{k} \\ 0 & p_{2^{k}}(i, j)<T_{k}\end{array}\right.$

Step 5. If the estimated radial components are $R^{*}(i, j)$ $=0$, set the angle $\theta, \phi$ equal to zero.

Step 6. The components $\theta, \phi, R^{*}$ in spherical coordinates do inverse spherical transform as follows:

$\left\{\begin{array}{l}\left(C_{j, k}^{(1)}\right)^{*}=R^{*} \sin \varphi \cos \theta \\ \left(C_{j, k}^{(2)}\right)^{*}=R^{*} \sin \varphi \sin \theta \\ \left(C_{j, k}^{(3)}\right)^{*}=R^{*} \cos \varphi\end{array}\right.$ 


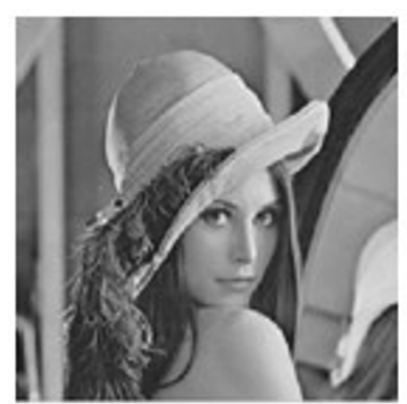

(a)

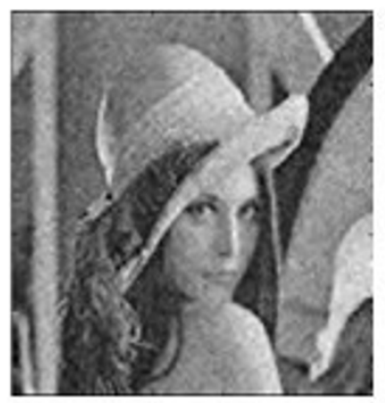

(e)

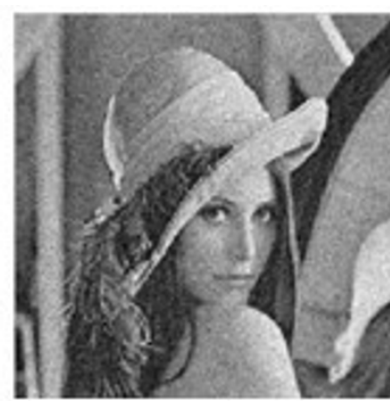

(b)

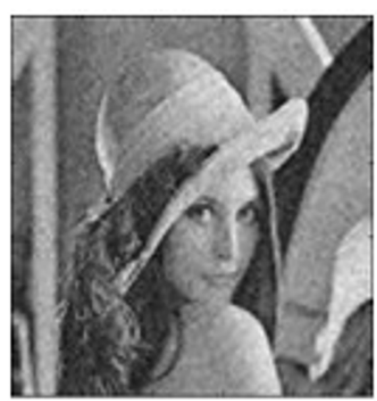

(f)

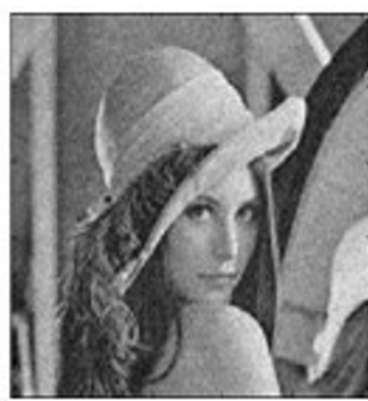

(c)

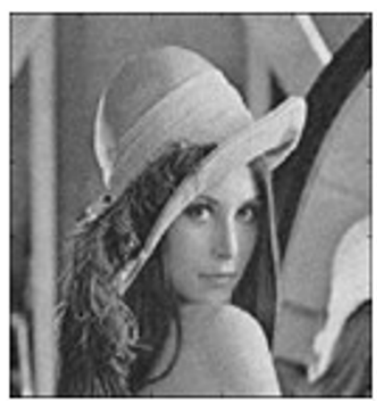

(g)

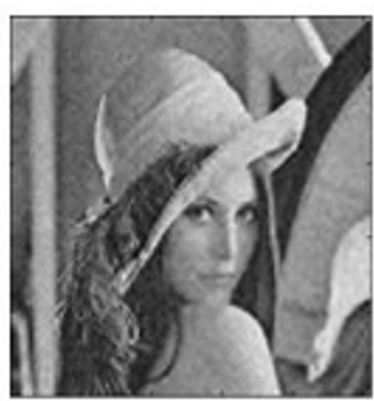

(d)

Figure 4 Image lena and experimental results. (a) Original image lena. (b) Gaussian white noising image. (c) Hyperbolic shrinkage in [15]. (d) MATS method in [14]. (e) Improved soft-threshold in [16]. (f) AdpMpMHard in [18]. (g) This article.

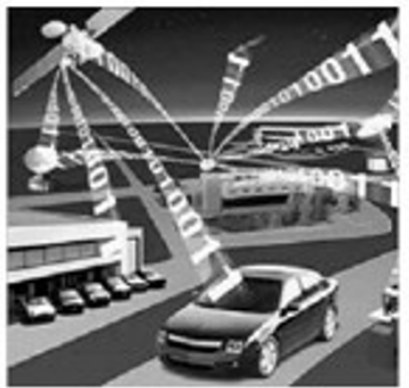

(a)

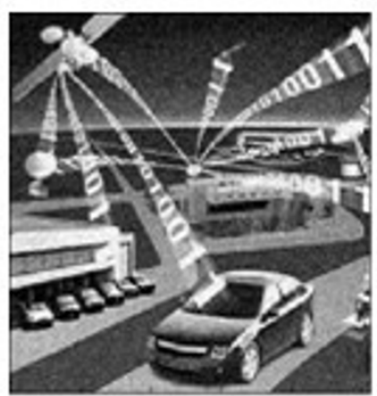

(e)

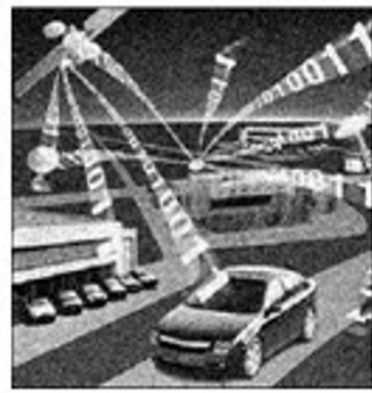

(b)

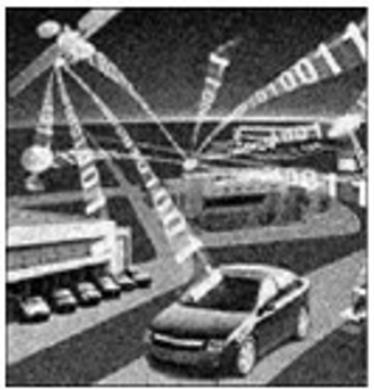

(f)

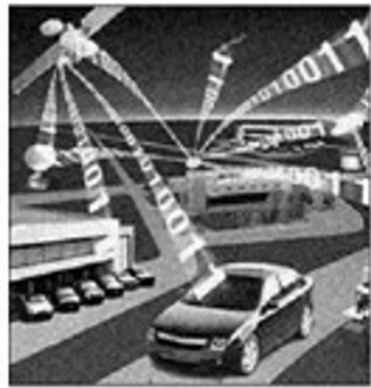

(c)

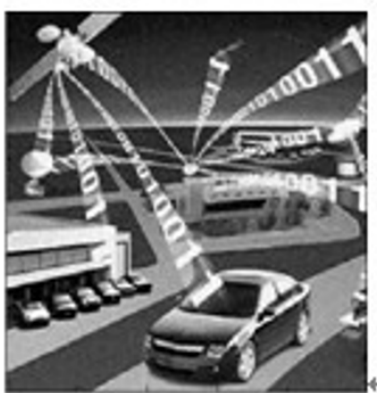

(g)

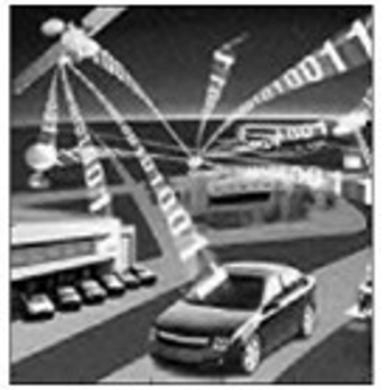

(d)

Figure 5 Image IOT and experimental results. (a) Original image IOT. (b) Gaussian white noising image. (c) Hyperbolic shrinkage in [15]. (d) MATS method in [14]. (e) Improved soft-threshold in [16]. (f) AdpMpMHard in [18]. (g) This article. 
where $\left(C_{j, k}^{(1)}\right)^{*},\left(C_{j, k}^{(2)}\right)^{*},\left(C_{j, k}^{(3)}\right)^{*}$ are estimated horizontal high frequency, vertical high frequency, and diagonal high frequency, respectively.

Step 7. Finally, to realize de-noising and reconstruct the image.

\section{Experiments}

To illustrate the effectiveness of this improved method, experimentation is done to use the image lena and the Internet of things (IOT) which sizes are $256 * 256$.

\subsection{The evaluation criteria}

This article evaluates the de-noising effect from the peak signal-to-noise ratio (PSNR), mean square error (MSE), and the runtime.

$$
\begin{aligned}
& \text { PSNR }=10 \log 10\left(255^{\wedge} 2 / \mathrm{MSE}\right) \\
& \mathrm{MSE}=\frac{1}{M N} \sum_{i=0}^{M-1} \sum_{j=0}^{N-1}\left(f_{i, j}^{*}-f_{i, j}\right)
\end{aligned}
$$

\subsection{The choice of decomposition level}

First, we must determine the decomposition levels. Table 1 shows the de-noising effects evaluation of different decomposing level. In this simulation experimentation, the improved algorithm is used for image denoising and Gaussian white noise (the standard deviation is 20) is added to image lena and IOT.

From Table 1, we can get the conclusion that the denoising effect is asymptotically stable after three levels. When the scale is greater than six levels, turbulence will generate in PSNR and MSE. Therefore, the best decomposition level is 3-5.

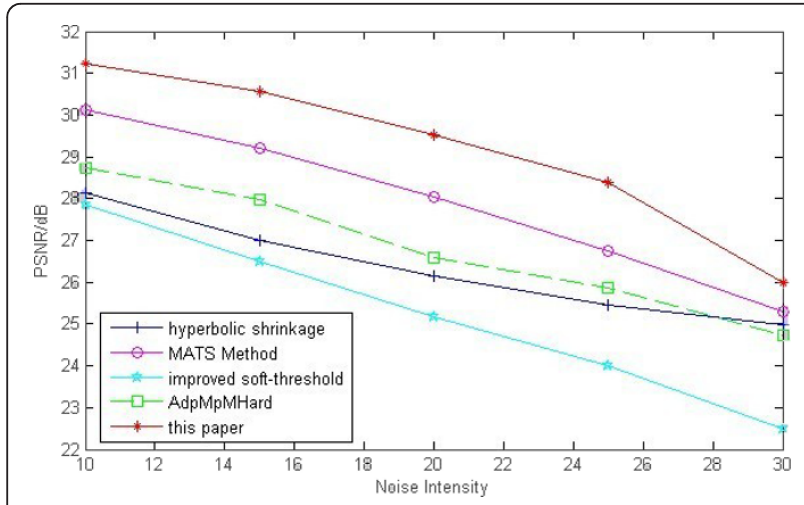

Figure 6 Comparison of PSNR for kinds of de-noising methods to Gaussian white noising image lena.

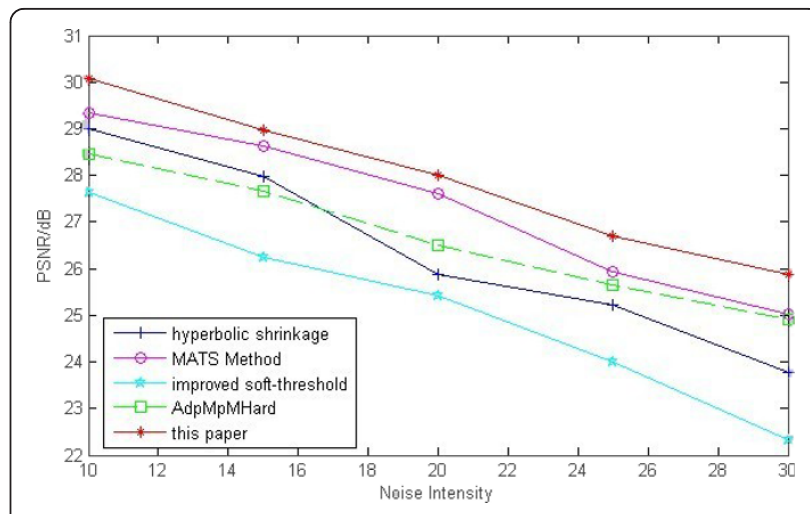

Figure 7 Comparison of PSNR for kinds of de-noising methods to Gaussian white noising image IOT.

\subsection{The de-noising results adding Gaussian white noise}

According to the above assessment, level 4 is an ideal decomposition level. Here, level $=4$, adding the Gaussian white noise (the standard deviation is 20). The denoising results of different methods are shown in Figures 4 and 5. Figure 4 shows the visual results of image lena. Figure 5 shows the visual results of image IOT. The results show that this proposed algorithm visually superior to other algorithms.

Figures 6 and 7 show the comparison of PSNR for kinds of de-noising methods to images lena and IOT. Figures 8 and 9 show the comparison of MSE for kinds of de-noising methods to images lena and IOT. From the comparisons of PSNR and MSE, we can get the conclusion that the proposed algorithm in this article has better de-noising effect.

\subsection{The comparison of running time}

Figure 10 demonstrates the run time of different denoising methods. It shows that the proposed algorithm retains the advantages of spherical coordinate system.

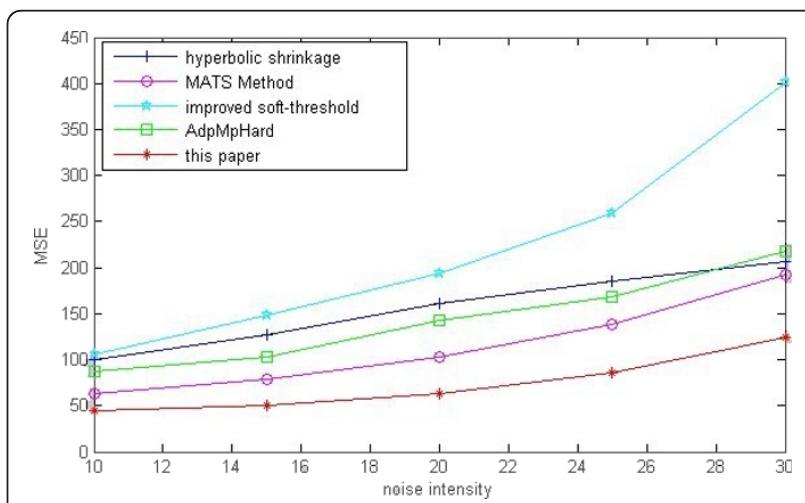

Figure 8 Comparison of MSE for kinds of de-noising methods to Gaussian white noising image lena. 


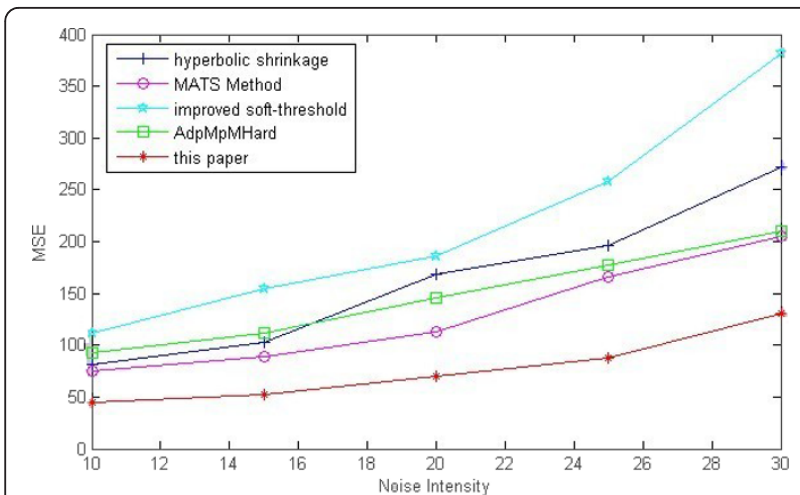

Figure 9 Comparison of MSE for kinds of de-noising methods to Gaussian white noising image IOT.

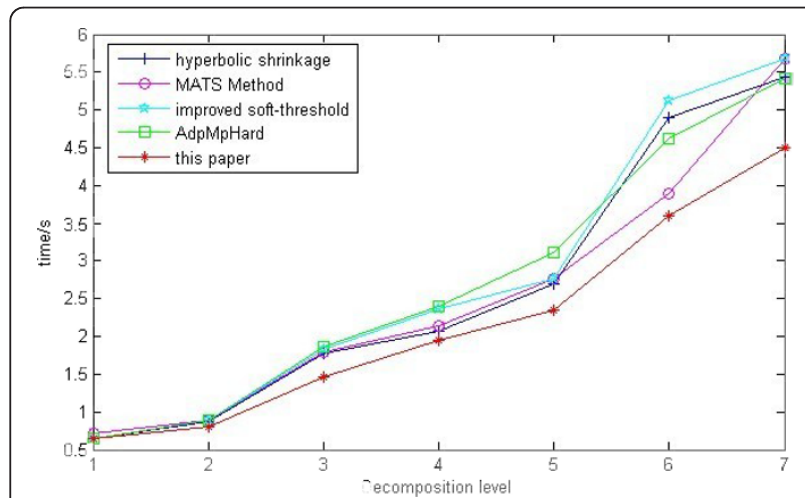

Figure 10 Run time evaluation for kinds of de-noising methods.

The running time is less than other algorithms. With the scale increase, this advantage is more obvious.

\subsection{The de-noising results adding salt\&pepper noise}

Gaussian white noise and salt\&pepper noise are the most common types of noise. Figure 11 shows the visual

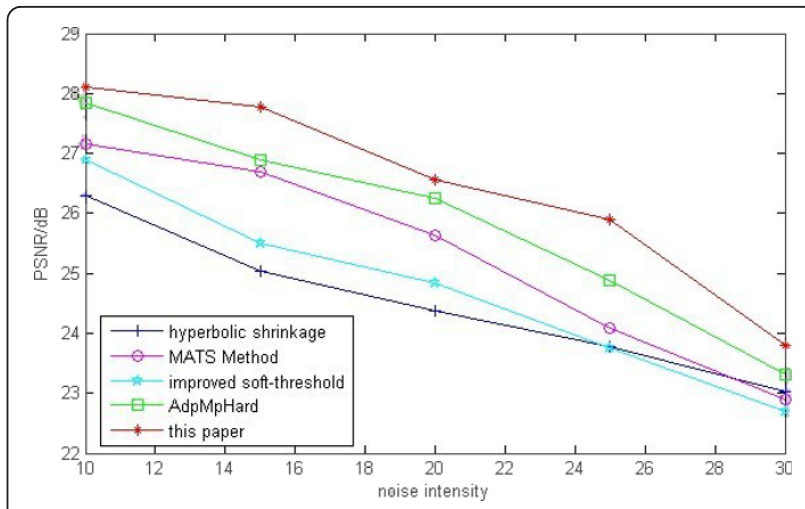

Figure 12 Comparison of PSNR for kinds of de-noising methods to salt\&pepper noising image lena.

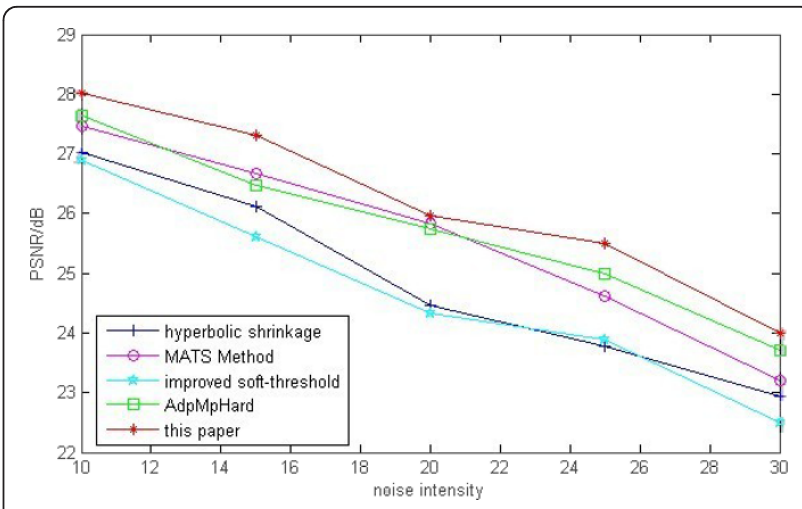

Figure 13 Comparison of PSNR for kinds of de-noising methods to salt\&pepper noising image lena.

de-noising results added in the salt\&pepper noise. According to Figure 11, the proposed method is also effective to salt\&pepper noising image.

Figures 12 and 13 demonstrate the comparison of PSNR for kinds of de-noising methods to salt\&pepper noising images lena and IOT. Compared with other

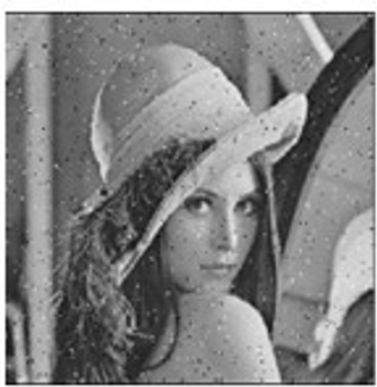

(a)

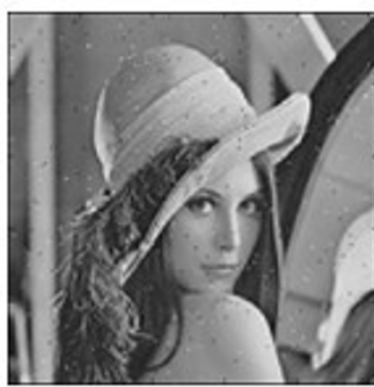

(b)

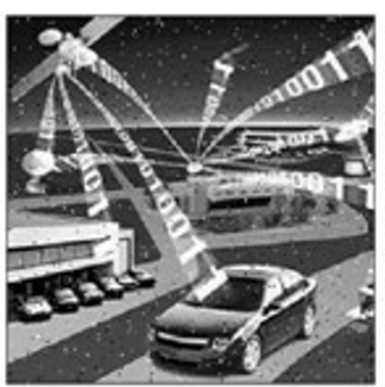

(c)

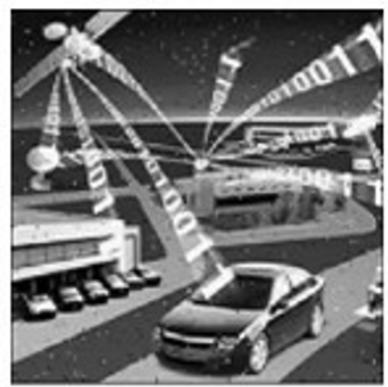

(d)

Figure 11 Salt\&pepper noising image and experimental results. (a) Salt\&pepper noising image lena. (b) De-noising result of this paper. (c) Salt\&pepper noising image IOT. (d) De-noising result of this article. 
algorithms, the de-noising results of proposed method have more advantages after adding salt\&pepper noise.

\section{Conclusion}

In this article, we use spherical coordinates system to remove the relevance of coefficients. Based on the Besov space norm theory, this article derives a new threshold in spherical coordinate system. MSNP is defined to make full use of the correlation in different decomposition scales. A new adaptive curve shrinkage function is constructed. This function can reach and exceed the true value. It largely avoids the edge blurry during image de-noising. The new threshold controls MSNP for image de-noising. Experimental results show that the proposed method visually superiors to other algorithms, and it is more effective in PSNR, MSE, and runtime.

\section{Acknowledgements}

This study was supported by the National Natural Science Foundation of China (Nos. 61170173, 60773073, and 61001174), the Program for New Century Excellent Talents in University of China (No. NCET-09-0895), the Key project of Ministry of Education of China (No. 208010), and the Tianjin Natural Science Foundation (No. 10JCYBJC00500)

\section{Author details}

${ }^{1}$ Key Laboratory of Computer Vision and System, Tianjin University of Technology, Ministry of Education, Tianjin 300384, China ${ }^{2}$ Tianjin Key Laboratory of Intelligent Computing \& Novel Software Technology, Tianjin University of Technology, Tianjin 300384, China

\section{Competing interests}

The authors declare that they have no competing interests.

Received: 28 December 2011 Accepted: 16 May 2012

Published: 16 May 2012

\section{References}

1. M Frandes, I Magnin, R Prost, Wavelet thresholding-based denoising method of list-mode MLEM algorithm for compton imaging. IEEE Trans Nuclear Sci. 58(3 Part 1), 714-723 (2011)

2. HO Yacov, D Shaked, A discriminative approach for wavelet denoising. IEEE Trans Image Process. 17(4), 443-457 (2008)

3. G Chen, S Qian, Denoising of hyper spectral imagery using principa component analysis and wavelet shrinkage. IEEE Trans Geosci Remote Sens. 49(3), 973-980 (2011)

4. D Zhang, A new approach and system for attentive mobile learning based on seamless migration. Appl Intell. 36(1), 75-89 (2012)

5. H Yu, L Zhao, H Wang, Image denoising using trivariate shrinkage filter in the wavelet domain and joint bilateral filter in the spatial domain. IEEE Trans Image Process. 18(10), 2364-2369 (2009)

6. D Zhou, Q Wang, Q Tian, Q Lin, W Fu, Wavelet analysis and its application in denoising the spectrum of hyperspectral image. Spectrosc Spectral Anal. 29(7), 1941-1945 (2009)

7. S Parrilli, M Poderico, CV Angelino, L Verdoliva, A nonlocal SAR image denoising algorithm based on LLMMSE wavelet shrinkage. IEEE Trans Geosci Remote Sensing. 50(2), 606-616 (2012)

8. D Zhang, X Zhang, A new service-aware computing approach for mobile application with uncertainty. Appl Math Inf Sci. 6(1), 9-21 (2012)

9. DL Donoho, Denoising by soft-thresholding. IEEE Trans Inf Theory. 41(3) 613-627 (1995)

10. DL Donoho, IM Johnstone, Ideal spatial adaptation via wavelet shrinkage. Biometrika. 81, 425-455 (1994)

11. RA DeVore, Nonlinear Approximation, (Cambridge University Press, London, 1998), pp. $51-150$
12. $\mathrm{X}$ Feng, $\mathrm{D}$ Jiang, $\mathrm{G} \mathrm{Xu}$, Combining variation and wavelet transform for image zooming. Chin J Comput. 31(2), 340-345 (2008)

13. A Chambolle, R DeVore, $N$ Lee, B Lucier, Nonlinear wavelet image processing: variational problems, compression, and noise removal through wavelet shrinkage. IEEE Trans Image Process. 7(3), 319-335 (1998)

14. A Khare, US Tiwary, W Pedrycz, M Jeon, Multilevel adaptive thresholding and shrinkage technique for denoising using Daubechies complex wavelet transform. Imag Sci J. 58(6), 340-358 (2010)

15. S Wang, Y Zhou, D Zou, Nonlinear wavelet image compression coding algorithms based on spherical coordinates. J Infrared Millimet Waves. 20(5), 387-390 (2001)

16. C Li, J Sun, Y Kang, Adaptive image denoising by a new thresholding function. WiCOM. 5600682, 1(1), 1-5 (2010)

17. $\quad P$ Bao, $L$ Zhang, Noise reduction for magnetic resonance images via adaptive multiscale products thresholding. IEEE Trans Med Imag. 22(9), 1089-1099 (2003)

18. J Meng, Q Pan, H Zhang, Optimal threshold determination for multiscale product in wavelet denoising. ISCIT. 1566924, 1(1), 570-573 (2005)

19. J Skowronski, A nonlinear transform for subband image coding. Signal Process. 77, 289-307 (1999)

doi:10.1186/1687-6180-2012-110

Cite this article as: Zhang et al: A novel image de-noising method based on spherical coordinates system. EURASIP Journal on Advances in Signal Processing 2012 2012:110

\section{Submit your manuscript to a SpringerOpen ${ }^{\circ}$ journal and benefit from:}

- Convenient online submission

- Rigorous peer review

- Immediate publication on acceptance

- Open access: articles freely available online

- High visibility within the field

- Retaining the copyright to your article

Submit your next manuscript at springeropen.com 